\title{
Cross-Racial Automatic Age Estimation from Facial Images using Deep Learning
}

\author{
Osekhonmen V. Abhulimen ${ }^{1}$, Akinwale O. Fadamiro ${ }^{2}$, Erastus O. Ogunti ${ }^{3}$ \\ ${ }^{123}$ Department of Electrical and Electronics Engineering, Federal University of Technology Akure, Nigeria. \\ ${ }^{1}$ bulioses@gmail.com, \\ 2 fadamiro@futa.edu.ng \\ 3 oguntig@gmail.com
}

Received Date : August 05, 2021 Accepted Date : August 27, 2021 Published Date : September 07, 2021

\begin{abstract}
This paper presents a deep learning approach for age estimation of human beings using their facial images. The different racial groups based on skin colour have been incorporated in the annotations of the images in the dataset, while ensuring an adequate distribution of subjects across the racial groups so as to achieve an accurate Automatic Facial Age Estimation (AFAE). The principle of transfer learning is applied to the ResNet50 Convolutional Neural Network (CNN) initially pretrained for the task of object classification and finetuning it's hyperparameters to propose an AFAE system that can be used to automate ages of humans across multiple racial groups. The mean absolute error of 4.25 years is obtained at the end of the research which proved the effectiveness and superiority of the proposed method.
\end{abstract}

Key words: Automatic Facial Age Estimation (AFAE), Convolutional Neural Network (CNN), Facial Aging Dataset (FAD), ResNet50,

\section{INTRODUCTION}

The intelligent interaction between humans and computers is of crucial importance to reach both the primary and contemporary goals of artificial intelligence (AI) [38] in age estimation. Face remains one of the most dominant and informative trait that is fundamental for human social interaction, identifying emotional tendencies, gender, health qualities and ethnicity or race [1]. Recent advancements in the field of Computer Vision in the last few decades shows, the human face has gradually become the focus of research in developing Biometric recognition systems. The appearance of a person's face is affected mostly by increase in age. The observation of aging-related features on faces allows humans to estimate the age of others just by looking at their face; but human based age estimation is in general less accurate when compared with identity and gender estimation [16]. As a result, Researchers in recent times have come up with a plethora of techniques for the extraction and use of these facial features in developing Automatic Facial Age Estimation (AFAE) systems. The relationship between facial appearance and aging is not quite linear, making it very complicated and varies for different persons [25]. This makes automatic facial age estimation whose primary objective is to be able to determine the age of a person using their facial images a very challenging topic as a result of the large facial appearance variations which is due to the combination of both extrinsic and intrinsic factors. Othmani et al [25] also revealed that among the various factors affecting aging and AFAE systems, race tend to possess the greatest challenge to human aging; as Africans or humans of Black racial group have unique aging features that cannot be learnt from other races. Notwithstanding, it is also an attractive topic due to its roots in many real-life applications, such as: health-care; Electronic Customer Relationship Management (ECRM), security control and surveillance, criminal investigation; age verification in sports; human computer interaction, job recruitment exercises, comparison between physiological age and actual age; web-content filtering and many other applications [2].

Though research has shown that automatic facial age estimation problems are very challenging; in recent years quality results are being obtained, but not much attention has been given to how distribution of subjects of different races in a facial aging dataset can impel or enhance the accuracy of facial age estimation systems used for age estimation tasks of humans across a variety of racial groups. This paper proposes a system for automatically estimating the age of humans via their facial images irrespective their race by using the deep learning approach based on ResNet50 Convolutional Neural Network (CNN).

\section{CLASSIFICATION OF RACIAL SKIN TYPES}

Generally, human race can be categorized into four broad groups based on skin colour. These are: Asian, Caucasian, Hispanic/Latino and Black [23].

It is important to note that each of these categories is heterogeneous, and the Asians, Caucasians and Hispanics/Latinos are be generally referred to as Light skin race in this paper. The awareness of these skin groups can help understand better how much the human skin colour affects the process of aging.

Pigmentation is the most obvious difference in skin characteristics between different racial groups [32]. This racial variation is dependent on the quantity of melanin, amount of ultra violet (UV) exposure, genetics, melanosome content and type of pigments found in the skin. Four chromophores are responsible for the varying colours found in human skin, these are: haemoglobin, oxyhaemoglobin, melanin and carotenoids [25]. Examining these racial skin colour types, [4] and [3] showed that the white pigmented skin types (Caucasians, Asians and 
Hispanics/Latinos) have approximately half as much epidermal melanin as the most darkly pigmented skin types (Blacks). However, the composition of white skin types was comparatively more enriched with lightly coloured alkali soluble melanin components. On an average, it has been found that stratum corneum from Black skin contained more corneocyte cell layers than that from other racial skin types, and the cell layers in Black skin were thought to be more compact perhaps reflecting greater intercellular cohesion [35]. Consistent with this, [14] found the mean electrical resistance of black skin types to be twice that of other racial skin colour, suggesting increased cohesiveness.

Research have shown that the lipid content of stratum corneum of black skin is higher than that of other racial skin types; and that trans-epidermal water loss (TEWL) which is the total amount of water vapor lost through the skin and appendages under non-sweating conditions of the skin is far greater in Black skin types as compared to the other racial skin types; also, water content (WC) in subjects with black skin colour is far lower than in subjects of other skin colour types [26], [35], [29] and [14].

Microscopic evaluation of skin [28] revealed that subjects with black skin contain larger mast cell granules than subjects with other skin colour types. The granules were 1.5 times larger and contained more parallel linear striations (PLS) and had 30\% less curved lamellae. Black subjects were also discovered to have a lower threshold to thermal temperature and heat compared with subjects of other races [25].

Skin pigmentation dictates many of the changes in skin associated with aging. Nevertheless, all skin types experience photoaging changes but the more darkly pigmented subjects show the dermatological signs of aging at a more advanced age compared with more white pigmented subjects. Skin wrinkling and sagging is a predominant problem of whiter skin types whereas mottled hyperpigmentation and uneven skin tone is associated with the darker skin types. This increased cohesivity may also explain the reduced potential to irritate black skin using a variety of chemical stimuli [11].

With these inherent underlying biological differences in racial skin types observed from these studies, it is clear that the Black race have very distinct characteristics from the other racial groups (Caucasians, Asians and Hispanics/Latinos). Hence their very distinct aging patterns that cannot be learnt from the other races as observed in this paper.

\section{RELATED WORKS}

Alot of research interest is been drawn in the field of AFAE due to its diverse application in solving real-world problems. Extraction of ageing patterns (feature extraction) from facial images manually, using a set of rules and algorithms is the first phase in AFAE involving handcrafted traditional machine learning based methods. These features have been categorized into: local, global and hybrid [21]. Anthropometric based models [17], Active Appearance Model (AAM) [6] as well as Bio-Inspired Features (BIF) created by Gabor filters [12] and Local Binary Patterns (LBP) [36] were also vastly used to learn texture and shape features from the training facial images.

In recent times, high-level semantic features are developed using deep learning methods based on CNNs in order to attain improved accuracy and efficiency in AFAE systems. The multi-level CNNs execute a chain of transformations on the facial image in order to learn a denser transformation representation of the facial image. Further abstract features or characteristics are learnt in the deeper layers which give rise to a more accurate estimation of the class of the ageing patterns. The high-level semantic features extracted by using deep learning methods outperform traditional hand-crafted features [5].

In [25], Yang et al. combined ordinal regression with a CNN they called ScatNet to extract facial aging features and predict the age of the facial image. They used PCA (principal component analysis) to minimize the feature dimension and lastly 3-layers of fully connected $\mathrm{CNN}$ to produce the final decision via category-wise rankers. It was tested on MORPH, Lifespan and FACES datasets, and produced good results.

In [27], Rothe et al. introduced a method known as DEX (Deep Expectation), to estimate the age group of humans from a single image, before estimating the exact age value. This appeared to be more accurate and faster than when trying to estimate the exact age from a wide age range. The deep age estimation method was able to estimate apparent age without making use of explicit facial features. The IMBD-WIKI (Internet Movie Database-What I Know Is), FGNET, MORPH2 and CACD datasets were used in their experiment and they achieved a state-of-the-art result.

In order to overcome the problem of optical and motion blurring real-time facial images in age estimation, [15] introduced a deep CNN model based on ResNet152. The model excludes any form of manual intervention while training the feature vector coefficients and weights classifier. The whole algorithm of their proposed model involves the recognition of face and eye regions by making use of Adaboost detector; redefining and in-plane rotation of the facial image in the region of interest; and then lastly, using the pre-trained ResNet152 model to redefine the face region and estimate the person's age. Their method was experimented on the PAL and MORPH2 datasets.

Taheri and Toygar [15] combines multistage learned features from a generic feature extractor, a trained CNN model and a selection of age-related handcrafted features. Two approaches were implemented in this method. The first approach involves a feature-level fusion of a variety of handcrafted local feature descriptors of facial components, skin and wrinkle; while the second approach involves score-level fusion of different feature vectors that were learned from multiple layers of a CNN used for age estimation. The method achieved meaningful results on the FGNET and MORPH2 datasets.

Mahjabin et al. [19] proposed a facial age estimator based on ResNet50 CNN architecture that implements age estimation majorly as a regression problem. Their methods involve a pre-processing phase which is made up of face detection, image resizing and one hot encoding. In order to improve the number of training data in the dataset, data augmentation approach such as flipping, rotating and zooming; were implemented. The performance of their method was tested on the FGNET dataset and training was done using the APPA-REAL and UTKFace datasets.

Fariza and Arifin [9] implemented a basic linear regression model architecture and a residual network classification approach based on ResNet50 and ResNeXt-50(34x4d) CNN to solve the problem of AFAE. The UTKFace database was used for testing the performance of the system.

Huynh and Nguyen, [13] proposed a system based on a Wide ResNet CNN for age and gender classification of certain Asian 
descents. Their approach involves an image augmentation stage which was done by using Random erasing and Mixup processing in order to improve the quality of the Megaage_Asian dataset and a training state, where the $\mathrm{CNN}$ was trained for age classification.

\section{METHODOLOGY}

Deep learning models are only as good as the data from which they are built, so a good data collection practice is crucial to developing high-performing AFAE system. Deep learning models are designed to automatically generate features, which saves feature engineering costs. Therefore, a larger amount of labelled data which should comprise of an adequate distribution of subjects of the four major racial groups in order for it to be more expressive [30] and of high accuracy was needed.

Dataset collection and dataset pre-processing are the first two major stages for developing the AFAE system.

\subsection{Data Collection and Pre-processing}

In this paper a Facial Aging dataset (FAE) composed of three datasets which were fused into one, was used. These are:

(i) UTKFace dataset [33]: The UTKFace dataset is a large unisex face database that contains over 23,000 facial images, with their ages spanning between $0-116$ years.

Though the UTKFace dataset can serve as an excellent benchmark for evaluating the performance of age estimation systems, there is a large disparity between the distribution of subjects across races. The dataset cuts across the four major races. While the Caucasians, Asians and Hispanics/Latinos (generally referred to as Whites) races share some close aging patterns, Blacks have some unique aging patterns that may not be learnt from other races [22]; hence their distribution in a cross-racial facial aging dataset should be given more preference. The distribution of Black subjects in the dataset is barely $20 \%$ of the total image population. So, in order have an optimum distribution (minimum of 25\%) of Black subjects in the dataset, and to actualize the aim of this research, the UTKFace dataset was augmented by complementing it with more facial images of subjects of Black race, from the APPA-REAL dataset and BlackFaces dataset.

(ii)APPA-REAL(ChaLearn LAP) database [8]: The Apparent and Real Age Estimation (APPA-REAL) facial image database consists of 7,591 facial images of both males and females.

Facial images of Black subjects were selected from this dataset to complement the population of Black subjects in the UTKFace dataset. The distribution of Black subjects in this dataset was observed to be less than $10 \%$ of the entire dataset population, which was still insufficient, to bring the population of Black subjects in the UTKFaces dataset to a minimum of $25 \%$.

(iii) BlackFaces dataset: This is a facial aging dataset created in the course of this research, between the period of March and July 2021; to further complement the distribution of Black subjects in the UTKFace dataset, due to the limited population of Blacks in the APPA-REAL dataset. It is a unisex facial image database, which contains 2,700 facial images of subjects solely of the Black race, with their ages ranging from 1-100 years.
The images were obtained by collecting real-time images of Black persons from the internet on social media websites like facebook.com, tinder.com, twitter.com and Google images as well as taking images of people with a digital camera. The image of each subject in the dataset is annotated with age, gender, date and time the image was collected. While collecting the images, it was ensured that they were frontal face images and that facial images with extreme facial expressions and additives (like excessive make-up and very obvious/dark face glasses) were avoided. This is the first dataset consisting solely of subjects of Black race, that can be used to solve the problem of AFAE.

After collecting these facial images, they were reannotated taking the same format as that of the images in UTKFace dataset, cropped and resized to $224 \times 224$ pixels using Harr Cascade classifier [34] and finally collated into a single folder, referred to as Facial Aging Dataset (FAD), before been fed to the CNN. The total number of images in the FAD used for training the CNN was 27,356. Figure 1 shows the pie chart of the UTKFace dataset before and after it was complemented with more facial images of Black subjects to create the Facial Ageing dataset (FAD) used for this research. It can be observed that the percentage of subjects of black racial group increased to $29 \%$ in the FAD. Figure 2 shows sample images from the FAD.

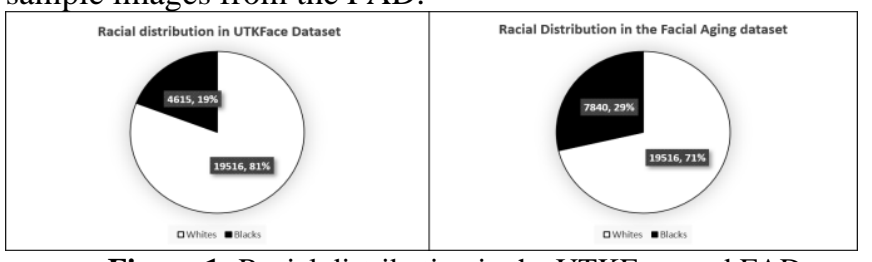

Figure 1: Racial distribution in the UTKFace and FAD.

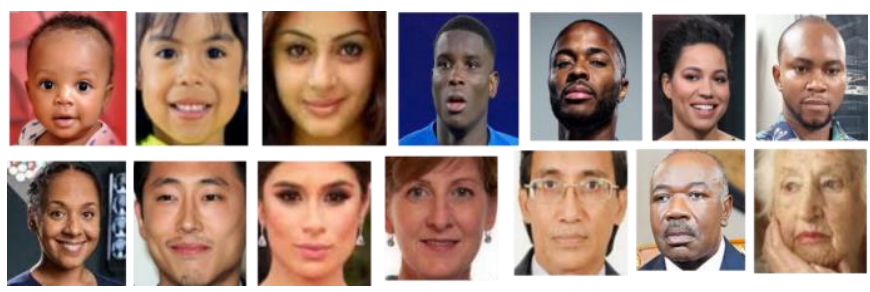

Figure 2: Sample images from the FAD

\subsection{Dataset split}

To effectively train the CNN, the Facial Aging Dataset (FAD) was first split into training and testing set by using a stratified cross validation (CV) approach defined by equation (1) and proposed by [10] that ensured an appropriate distribution of all ages in each of the training and testing set. $80 \%$ of the data was set aside for training the $\mathrm{CNN}$ while $20 \%$ for testing.

$$
C V(\hat{f})=\frac{1}{N} \sum_{i=1}^{N} L\left(y i, \hat{F}^{-\kappa(i)}\left(x_{i}\right)\right)
$$

where;

$L=$ loss function,

$-\kappa(i)$ means that the model $\hat{F}$ is trained without the training patterns in the same partition of the dataset as pattern $i$.

During training, $90 \%$ of the training set was used for learning the weights and $10 \%$ was used for validation. 
Osekhonmen V. Abhulimen et al., International Journal of Emerging Trends in Engineering Research, 9(9), September 2021, 1288 - 1294

\subsection{ResNet50 CNN Architecture}

For the deep learning task of cross-racial AFAE in this paper, the ResNet50 CNN [37] initially pretrained for image classification task on the ImageNet dataset [7] was used. It is a variant of the residual network model. with 48 Convolution layers along with 1 MaxPool and 1 Average Pool layer; consisting of 5 stages, each with a convolution and Identity block, having 3 convolution layers each; as shown in Figure 3. It relies on residual learning process, designed to solve complex visual tasks using more deeper layers stacked up together.

With ResNet50 CNN, it possible to train ultra-deep neural networks with hundreds or thousands of layers and still achieve great performance, because it utilizes the principle of skip connections and identity mapping which helps it to tackle the problem of gradient vanishing while still maintaining a high accuracy during training, with these it is;

(i) easier to train;

(ii) more tolerant of hyperparameters, including regularization and initial learning rate; and

(iii) generalizes better.

Hence the reasons for its choice over other CNN architectures in this paper.

The building block for ResNet50 CNN is defined with equation (2), while Figure 4 shows the diagram.

$y=\mathscr{F}^{*}\left(x,\left\{W_{i}\right\}\right)+x$

where $x$ and $y$ are the input and output vectors of the layers considered;

$y=\mathcal{F}^{*}\left(x,\left\{W_{i}\right\}\right)$ represents the residual mapping to be learned;

this is unlike other CNNs that can be seen as learning a function $y=\mathscr{F}^{\prime}(x)$.

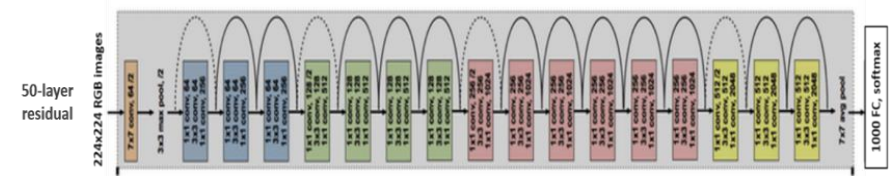

Figure 3: ResNet50 CNN Architecture

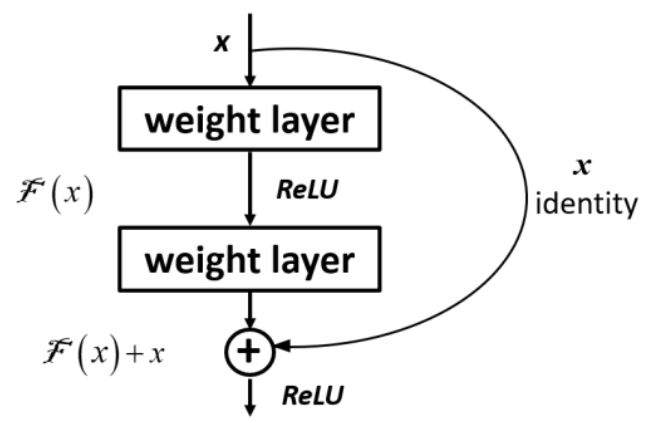

Figure 4: Building block of ResNet50 (identity mapping) [37]

\subsection{Fine-tunning the ResNet50 CNN with the FAD for AFAE task}

In fine-tunning the ResNet50 CNN for the task of AFAE, the following hyperparameters shown in Table 1 were used.

Table 1: ResNet50 CNN Hyperparameters

\begin{tabular}{c|c} 
Image size & $224 \times 224$ \\
Colour mode & RGB \\
Batch size & 64 \\
Random state & 42 \\
Optimizer(s) & SGD \\
Learning rate & 0.0001 \\
Epoch & 20 \\
Softmax layer & 116 \\
Frozen layers & $0 \%$
\end{tabular}

For evaluating the performance of the trained ResNet50 CNN for AFAE, the mean absolute error (MAE) was used as a performance metrics. It is defined with equation 3 .

$M A E=\frac{1}{n} \sum_{i=1}^{n}\left|\hat{y}_{i}-E_{i}(O)\right|$

where $n$ denotes the number of images used for testing; $\hat{y}$ is the real or actual age of a subject $i$; and $E_{i}(O)$ is the estimated age of that same subject. The lower the MAE value, the better the perfomance of the system.

\section{RESULTS AND DISCUSSIONS}

The images from the test set of the FAD were used to ascertain the performance of the age estimation system.

The model loss curve which shows the efficiency of the ResNet50 CNN model fine-tunned for the cross-racial facial age estimation task in this paper in learning necessary features needed for age estimation from the input dataset is shown in Figure 5. From the curve it is observed that the model learns at optimum level, without overfitting nor underfitting. Training is stopped at the $20^{\text {th }}$ epoch, to avoid overfitting, attaining a MAE of 4.25 years.

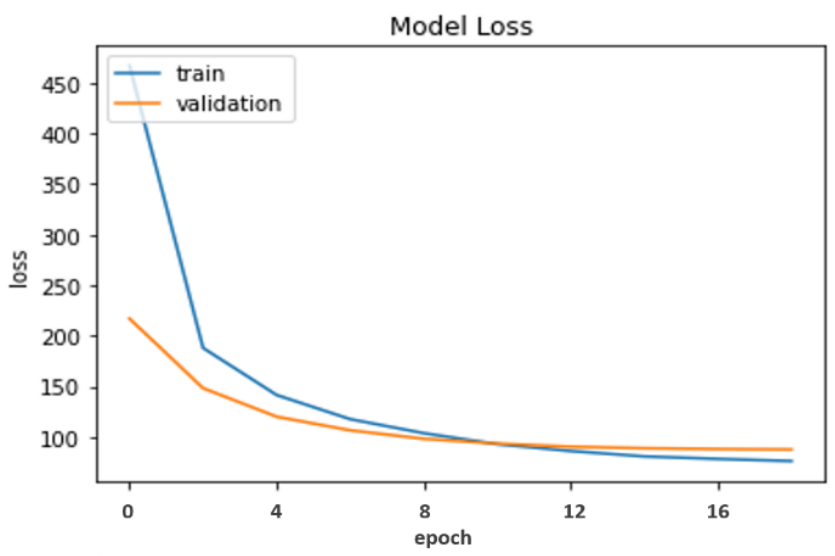

Figure 5: Model loss curve of train and validation dataset: loss vs epoch 
Images of Figure 6(a) and 6(b), show samples of subjects with their estimated ages alongside their actual ages performed by the proposed cross-racial AFAE system.

From the sample images of Figures 6(a) and 6(b) which consists of cross-racial subjects, it can be observed that the age disparity between the estimated and actual age of each subject lies approximately between the range of \pm 4 years; which corresponds with the MAE of 4.25 years attained and used as a benchmark in this paper.

\subsection{Performance Comparison}

To prove the superiority of the proposed system in estimating the ages of humans across race with emphasis on subjects of Black race, because of their distinct aging patterns that cannot be learnt from other races; the UTKFace dataset was also used in training the fine-tunned ResNet50 CNN, and a MAE of 5.37 years was obtained. The sample images of Figures 7(a) and 7(b) shows how well it was able to estimate the ages of subjects across race, in comparison with the proposed system.

From the sample images of Figures 7(a) and 7(b), it can be observed that though both systems gave almost a similar estimation of subjects of White race, the proposed system gave a better accuracy in estimating the ages of subjects of Black race.
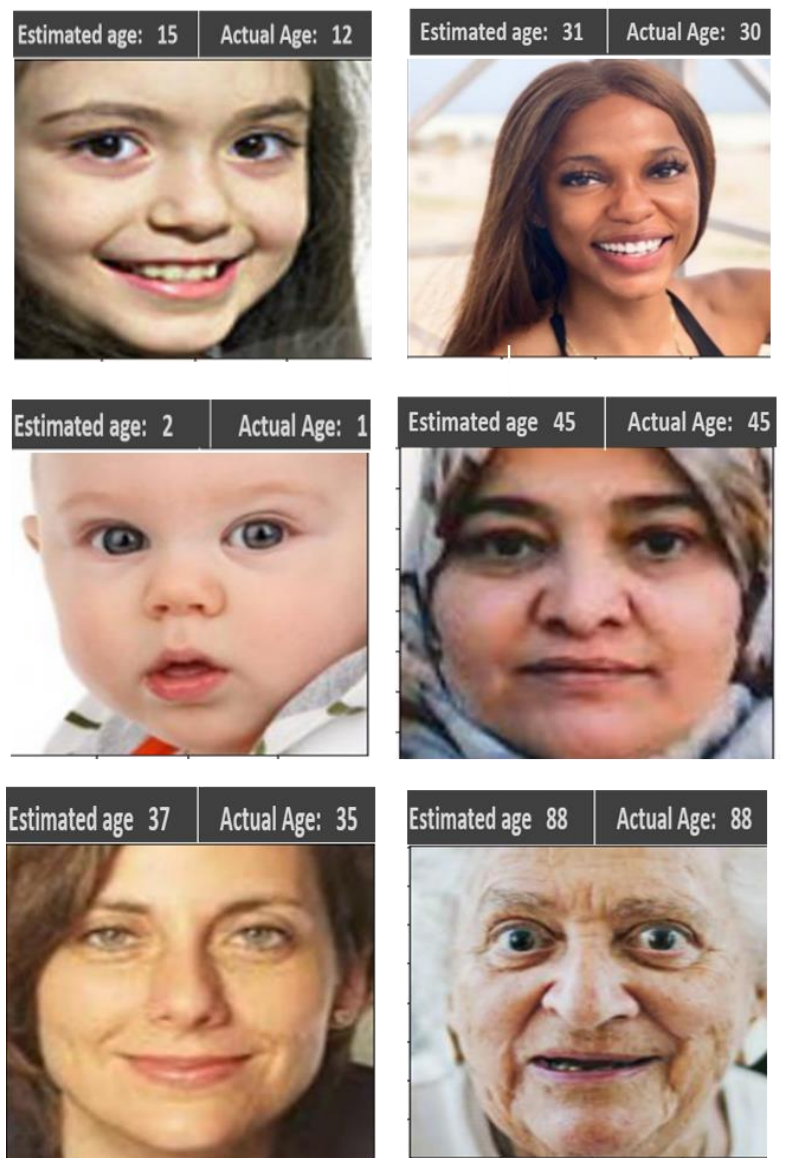

Figure 6(a): Sample images Subjects across the four racial groups with their estimated and actual ages

\subsection{Computational Complexity}

In the experimentation phase of this research, the recently developed ktrain library [20], which is an open-source library in Python, was used for building and training $\mathrm{CNN}$ on a $12 \mathrm{~GB}$ memory NVIDIA Telsa K80 GPU.

\section{CONCLUSION}

In this paper, a system that can estimate the ages of subjects across the four major multi-racial groups based on skin colour has been developed. This proposed system gave a better estimation of the ages of subjects across these racial groups, most significantly Black subjects, when compared to that trained solely with the UTKFaces dataset. This empirically brings to conclusion that the distribution of subjects of Black races should be balanced or adequately distributed to at least $25 \%$ of the total images in a multi-racial facial aging dataset, because they have distinct aging patterns that cannot be learnt from images of subjects of Caucasian, Asian and Hispanic/Latino races (generally referred to as Whites).
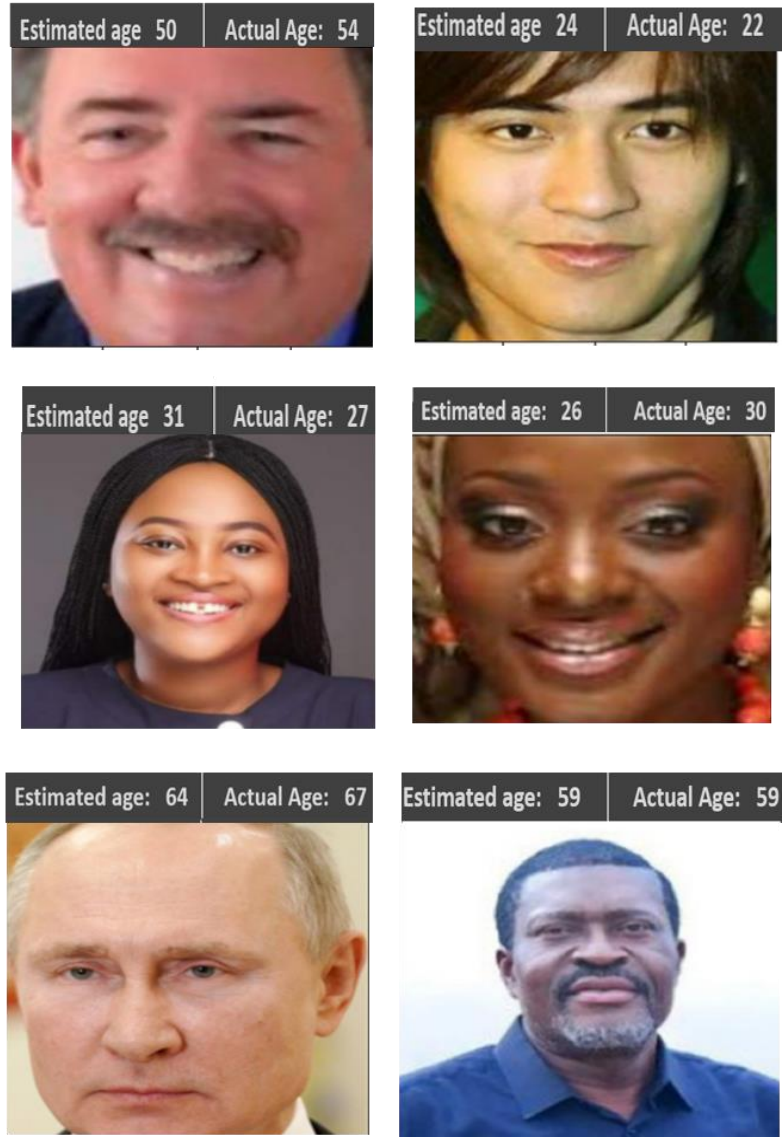

Figure 6(b): Sample images Subjects across the four racial groups with their estimated and actual ages. 


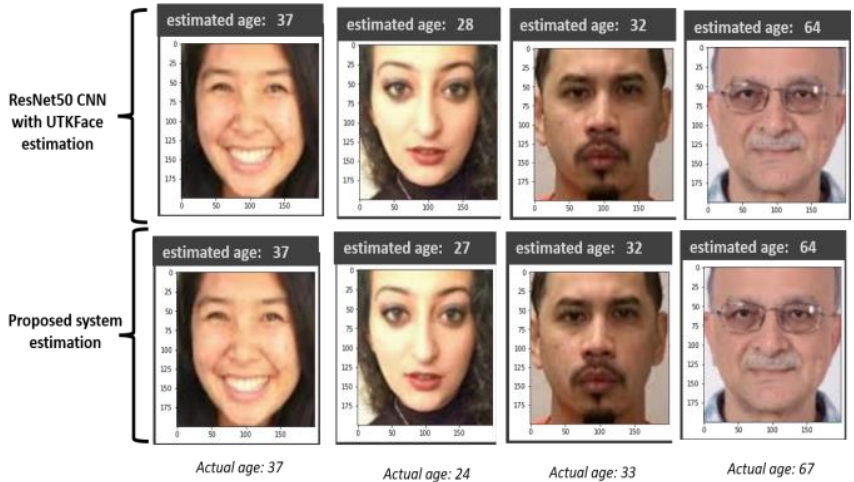

Figure 7(a): Comparison of the performance of the ResNet50 CNN trained with UTKFace dataset and the proposed system on subjects of White racial group.

\section{REFERENCES}

[1] D.A. Harris, S.A. Hayes-Skelton and V.M. Ciaramitaro. What's in a Face? How Face Gender and Current Affect Influence Perceived Emotion. Frontiers in Psychology, Vol. 7, p. 1468, 2016.

[2] O. Abhulimen and E. Ogunti. Facial Age Estimation using Deep Learning: A Review. Journal of Multidisciplinary Engineering Science and Technology (JMEST), Vol. 8, Issue 5, pp. 13927-13946, 2021.

[3] S. Alaluf, D. Atkins, K. Barrett, M. Blount, N. Carter and A. Heath. Ethnic variation in melanin content and composition in Photo-exposed and Photo-protected Human Skin. Pigment Cell Research. Vol. 15, pp. 112-118, 2002.

[4] S. Alaluf, A. Heath, N.I.K. Carter, D. Atkins, H. Mahalingam, K. Barrett and N. Smit. Variation in Melanin Content Composition in Type $\mathrm{V}$ and VI Photo-exposed and Photo-protected Human Skin: The Dominant role of DHI. Pigment Cell Research. Vol. 14, No. 5, pp. 337-347, 2001.

[5] M.M. Badr, A.M. Sarhan and R.M. Elbasiony. Facial Age Estimation using Deep Neural Networks: A Survey. In 2019 15th International Computer Engineering Conference (ICENCO), pp. 183-191, 2019.

[6] T. F. Cootes, G. J. Edwards and C. J. Taylor, Active appearance models. In IEEE Transactions on Pattern Analysis and Machine Intelligence, vol. 23, no. 6, pp. 681-685, 2001.

[7] J. Deng, W. Dong, R. Socher, L. Li, Kai Li and Li Fei-Fei. ImageNet: A large-scale hierarchical image database. In the IEEE Conference on Computer Vision and Pattern Recognition. Pp. 248-255, 2019.

[8] S. Escalera, X. Baró, H. J. Escalante and I. Guyon. ChaLearn looking at people: A review of events and resources. 2017 International Joint Conference on Neural Networks (IJCNN), pp. 1594-1601, 2017.

[9] A. Fariza and A.Z. Arifin. Age Estimation System Using Deep Residual Network Classification Method. In International Electronics Symposium (IES). Pp. 607-611, IEE, 2019.

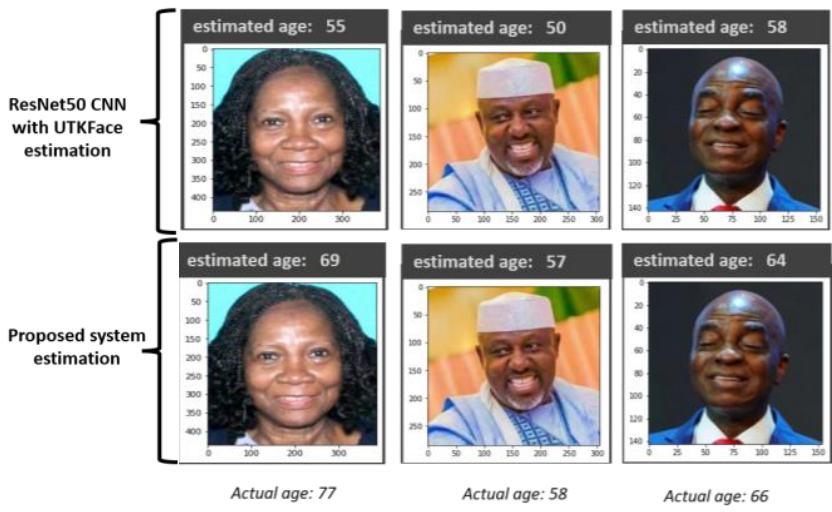

Figure 7(b): Comparison of the performance of the ResNet50 CNN trained with UTKFace dataset and the proposed system on subjects of Black racial group

[10] J. Friedman, T. Hastie, and R. Tibshirani. The Elements of Statistical Learning. Vol. 1, No. 10. New York: Springer series in statistics, 2001.

[11] P. Grimes, B.L. Edison, B.A. Green and R.H. Wildnauer. Evaluation of Inherent Differences Between African American and White Skin Surface Properties using Subjective and Objective Measures. Cutis. Vol. 73, No. 6, pp. 392-396, 2004.

[12] H. Han, C. Otto and A.K. Jain. Age Estimation from Face Images: Human vs. Machine Performance. In The International Conference on Biometrics (ICB). Pp. 1-8. IEEE, 2013.

[13] H.T. Huynh and H. Nguyen. Joint Age Estimation and Gender Classification of Asian Faces Using Wide ResNet. SN Computer Science. Vol 1, No. 5, pp. 1-9, 2020.

[14] L.C. Johnson, and N.L. Corah. Racial Differences in Skin Resistance. Science. Vol. 139, pp. 766-769, 1963.

[15] J.S. Kang, C.S. Kim, Y.W. Lee, S.W. Cho and K.R. Park. Age Estimation Robust to Optical and Motion Blurring by Deep Residual CNN. Symmetry, Vol. 10, No. 4, pp. 108, 2018.

[16] M.A. Khan. Automatic Facial Age Estimation. Ph.D dissertation, Lancaster University, School of Computing and Communications, United Kingdom,

2015.

[17] Y.H. Kwon and N. da Vitoria Lobo. Age Classification from Facial Images. Computer Vision and Image Understanding. Vol. 74, No. 1, pp. 1-21, 1999.

[18] D. Larsen-Freeman, D. Transfer of Learning Transformed. Language Learning. Vol. 63, pp.107-129, 2013.

[19] S. Mahjabin, M.M. Alam and K.H. Talukder. Age Estimation from Facial Image Using Convolutional Neural Network (CNN). In The Proceedings of International Conference on Computer, Communication, Chemical, Materials and Electronic Engineering (IC4ME2). Pp. 1-4, IEEE, 2019.

[20] A.S. Maiya. ktrain: A Low-Code Library for Augmented Machine Learning. 2020. Available at arXiv:2004.10703 [cs.LG]. 
[21] O.F. Osman and M.H. Yap. Computational Intelligence in Automatic Face Age Estimation: A Survey. IEEE Transactions on Emerging Topics in Computational Intelligence. Vol 3, No. 3, pp. 271-285, 2018.

[22] A. Othmani, A.R. Taleb, H. Abdelkawy and A. Hadid. Age Estimation from Faces using Deep Learning: A Comparative Analysis. Computer Vision and Image Understanding. Vol. 196, pp. 1-11, 2020.

[23] Peptan: Does Ethnicity Affect How our Skin Ages? 2021.

Available

at

https://www.peptan.com/does-ethnicity-affect-how-ourskin-ages/

[24] H.F. Yang, B.Y. Lin, K.Y. Chang and C.S. Chen. Automatic Age Estimation from Face Images via Deep Ranking. Networks. Vol. 35, No. 8, pp. 1872-1886, 2013.

[25] A.V. Rawlings. Ethnic Skin Types: Are there Differences in Skin Structure and Function? International Journal of Cosmetic Science. Vol. 28, No. 2, pp. 79-93, 2006.

[26] R.P. Rienertson and V.R. Wheatley. Studies on the Chemical Composition of Human Epidermal Lipids. Journal of Investigative Dermatology. Vol. 32, pp. 49-69, 1959.

[27] R. Rothe, R. Timofte and L. Van Gool, L. DEX: Deep Expectation of apparent age from a single image. In Proceedings of the IEEE International Conference on Computer Vision Workshops. Pp. 10-1, 2015.

[28] H. Sueki, D. Whitaker-Menezes and A.M. Kligman. Structural Diversity of Mast cell Granules in Black and White Skin. British Journal of Dermatology. Vol. 144, No. 1, pp. 85-93, 2001.

[29] K. Sugino, G. Imokawa and H.I. Maibach. Ethnic Difference of Stratum Corneum Lipid in Relation to Stratum Corneum Function. Journal of Investigative Dermatology. Vol. 100, p. 587, 1993.

[30] K. Sveikata, I. Balciuniene and J. Tutkuviene. Factors Influencing Face Aging. Literature Review. Stomatologija, Vol. 13, No. 4, pp. 113-116, 2011.

[31] S. Taheri and Ö. Toygar. Multi-stage Age Estimation using Two Level Fusions of Handcrafted and Learned Features on Facial Images. IET Biometrics. Vol. 8, No. 2, pp. 124-133, 2018.

[32] S. Taylor. Understanding Skin of Colour. Supplementary Journal of the American Academy Dermatology. Vol. 46, pp. 41-42, 2002.

[33] UTKFace. Large Scale Face Dataset. 2017. Available at https://susanqq.github.io/UTKFace/

[34] P. Viola and M. Jones. Rapid Object Detection using a Boosted Cascade of Simple Features. In Proceedings of the IEEE Computer Society Conference on Computer vision and Pattern Recognition (CVPR 2001). Vol. 1, pp. 1-11. IEEE, 2001.

[35] D.A. Weigand, C. Haygood and J.R. Gaylor. Cell Layer and Density of Negro and Caucasian Stratum Corneum. Journal of Investigative Dermatology. Vol. 62, No. 6, pp. 563-568, 1974.
[36] A. Gunay and V. V. Nabiyev. Automatic age classification with LBP. 2008 23rd International Symposium on Computer and Information Sciences. Pp. 1-4. 2008.

[37] K. He, X. Zhang, S. Ren and J. Sun. Deep residual learning for image recognition. In Proceedings of the IEEE conference on computer vision and pattern recognition. Pp. 770-778, 2016.

[38] S. Singh, D. Singh and V. Yadav. Face Recognition using HOG Feature Extraction and SVM Classifier. International Journal of Emerging Trends in Engineering Research. Vol. 8, No. 9, pp. 6437-6440, 2020. 